\title{
Reactivation of Epstein-Barr virus lytic cycle by histone deacetylase inhibitors
}

\author{
Kwai Fung Hui ${ }^{1,2}$, Alan Kwok Shing Chiang ${ }^{1,2}$ \\ ${ }^{1}$ Department of Paediatrics and Adolescent Medicine, Li Ka Shing Faculty of Medicine, The University of Hong Kong, Queen Mary \\ Hospital, Pokfulam, Hong Kong SAR, China \\ ${ }^{2}$ Center for Nasopharyngeal Carcinoma Research, The University of Hong Kong, Hong Kong SAR, China
}

Correspondence: Alan KS Chiang

E-mail: chiangak@hkucc.hku.hk

Received: September 22, 2015

Published online: November 02, 2015

\begin{abstract}
Epstein-Barr virus (EBV) is closely associated with certain lymphoid and epithelial malignancies such as Burkitt lymphoma, nasopharyngeal carcinoma (NPC) and gastric carcinoma (GC). In the tumor cells, the virus persists in a tight latency, expressing a limited number of latent proteins. Reactivation of EBV lytic cycle from latency leads to expression of many more viral lytic proteins which may provide potential therapeutic targets for the EBV-associated cancers. Histone deacetylase (HDAC) inhibitors belong to an emerging class of anti-cancer agents which work through acetylation of different histone and non-histone proteins in cancer cells. Our previous work showed that various pan-HDAC inhibitors, which inhibit eleven HDAC isoforms, can preferentially reactivate EBV lytic cycle in EBV-positive epithelial rather than lymphoid cancers and mediate enhanced killing of EBV-positive NPC and GC cells through augmentation of apoptotic cell death. Recently, we found that a selective class I HDAC inhibitor, romidepsin, can potently induce EBV lytic cycle in NPC and GC cells and confer susceptibility of the induced cells to killing by an anti-viral agent, ganciclovir, in vitro and in vivo. The reactivation of $\mathrm{EBV}$ lytic cycle by romidepsin is related to the inhibition of $\mathrm{HDAC}-1,-2$ and -3 isoforms and the activation of PKC- $\delta$. Interestingly, our current findings suggest that acetylation of non-histone proteins might also play a role in the regulation of EBV lytic cycle upon HDAC inhibition. In this review, we discuss our recent findings on the mechanisms of EBV lytic cycle reactivation and propose possible strategies in using HDAC inhibitors for the treatment of EBV-associated cancers.
\end{abstract}

Keywords: Romidepsin; histone deacetylase inhibitor; Epstein-Barr virus; epithelial cancer; lytic cycle reactivation

To cite this article: Kwai Fung Hui, et al. Reactivation of Epstein-Barr virus lytic cycle by histone deacetylase inhibitors. Can Cell Microenviron 2015; 2: e1033. doi: 10.14800/ccm.1033.

\section{Introduction}

Epstein-Barr virus (EBV) is a ubiquitous gamma herpesvirus which infects more than $90 \%$ of populations worldwide. It is closely associated with several types of lymphomas (e.g. endemic Burkitt lymphoma, Hodgkin lymphoma, nasal NK/T-cell lymphoma and post-transplant lymphoproliferative disorder) and epithelial malignancies (nasopharyngeal carcinoma [NPC] and gastric carcinoma $[\mathrm{GC}])^{[1,2]}$. EBV has a biphasic life cycle, namely latent and lytic cycles. In the EBV-associated cancers, the virus persists in the latent cycle in every tumor cell and expresses a very limited number of viral latent proteins. Induction of EBV from its latent to lytic cycle can lead to the sequential expression of a series of lytic proteins, namely the immediate early (e.g. Zta or Rta), early (e.g. BMRF1 or BGLF1) and late lytic proteins (e.g. VCA-p18 and gp350/220). The expression of more viral lytic proteins will lead to higher immunogenicity and provide an opportunity for therapeutic targeting against the EBV-associated cancers. 
Oncolytic therapy, which involves pharmacological reactivation of the latent virus into lytic cycle to confer susceptibility of EBV-infected cells to antiviral drugs, is being explored as a potential therapeutic strategy against the EBV-associated diseases ${ }^{[3,4]}$. Studies have shown that ganciclovir (GCV), a nucleoside-type antiviral drug, can enhance tumor cell death in combination with different types of lytic inducers. Since the killing effect of GCV relies on expression of viral lytic protein kinase BGLF4 ${ }^{[5]}$, the efficiency and specificity of lytic induction greatly impact the efficacy of this treatment strategy. Understanding the mechanisms controlling the lytic reactivation is important for the development of specific and potent lytic inducing agents for the treatment of EBV-associated cancers.

The summative action of histone acetyltransferases (HATs) and histone deacetylases (HDACs) regulates lysine acetylation of different cellular proteins. HATs transfer acetyl groups from acetyl-coenzyme A to $\varepsilon$-amino groups of lysine residues in the $\mathrm{N}$-terminal tails of proteins whilst HDACs remove the acetyl groups from the lysine residues of proteins [6]. Lysine acetylation of histone proteins plays an important role in regulation of gene expression because it neutralizes the positive charge of histone and results in an open chromatin structure for expression of genes associated with the acetylated histones [7, 8]. It has been shown that acetylation of histones on the immediately-early promoter of human cytomegalovirus ${ }^{[9]}$ and BRLF1 promoter of EBV ${ }^{[10]}$ can result in reactivation of viral lytic cycle in the virus-associated cancers. On the other hand, lysine acetylation can also directly regulate the activities of various non-histone protein substrates ${ }^{[11,12]}$. A recent study identified 3600 acetylation sites in 1750 cellular proteins in different human cancer cell lines using a high resolution mass spectrometry analysis, suggesting the possibility of previously unexplored roles of protein acetylation in various cellular processes ${ }^{[12]}$.

Pan-HDAC inhibitors such as trichostatin A or suberoylanilide hydroxamic acid (SAHA) are able to inhibit class I (HDAC1/2/3/8), class II (HDAC4/5/6/7/9/10) and class IV (HDAC11) but not NAD+ dependent class III HDAC isoforms ${ }^{[7]}$. We have reported that pan-HDAC inhibitors, such as trichostatin A, sodium butyrate, valproic acid and SAHA can reactivate lytic cycle of EBV and mediate enhanced killing of EBV-positive epithelial cancer cells, including NPC and GC ${ }^{[13,14]}$. Based on the potent effect of HDAC inhibitors on the reactivation of viral lytic cycle in EBV-positive epithelial cancer cells, we endeavored to investigate the mechanisms by which HDAC inhibitors reactivate EBV lytic cycle as essential steps to translate the use of HDAC inhibitors for the clinical treatment of EBV-positive epithelial malignancies. We tested the hypothesis that inhibition of several specific HDAC isoforms by selective HDAC inhibitors can potently reactivate EBV lytic cycle in EBV-positive NPC and GC cells ${ }^{[15]}$. Our data showed that inhibition of HDAC-1, -2 and -3 by selective HDAC inhibitors such as romidepsin is sufficient to induce EBV lytic cycle in NPC and GC cells and confer susceptibility of the induced cells to killing by an anti-viral agent, ganciclovir, in vitro and in vivo ${ }^{[15]}$. These data have demonstrated an important role of HDAC inhibition in the regulation of lytic cycle reactivation and supported further investigation into the application of selective HDAC inhibitors such as romidepsin for the treatment of EBV-associated cancers. Our on-going work demonstrates, for the first time, that acetylation of non-histone proteins is involved in the lytic cycle reactivation by HDAC inhibitors in EBV-positive epithelial malignancies.

\section{Results and Discussion}

Our recent publication showed that a class I selective HDAC inhibitor, romidepsin, can potently induce EBV lytic cycle in EBV-positive epithelial malignanices including NPC and GC cells in vitro and in vivo ${ }^{[15]}$. When combined with ganciclovir (GCV), enhanced killing could be observed in the EBV-positive, but not EBV-negative NPC and GC cells ${ }^{[15]}$. Romidepsin is an FDA-approved drug for the treatment of cutaneous T-cell lymphoma and peripheral T-cell lymphoma ${ }^{[16]}$. The concentration of romidepsin which induces EBV lytic cycle $(\sim 5 \mathrm{nM})$ is much lower than the clinically achievable concentration in patients' plasma $(\sim 800$ $\mathrm{nM})^{[17]}$. These data support the use of romidepsin as a potent and safe lytic inducer of viral lytic cycle for the oncolytic therapy in EBV-associated epithelial cancers. The concept of the lytic induction therapy using combination of HDAC inhibitors, gemcitabine and GCV has been demonstrated to be work in clinical trials carried out in NPC patients ${ }^{[3,4]}$. It would be interesting to substitute valproic acid with romidepsin in such clinical trials. We predict that enhanced clinical outcomes can be observed in NPC patients because the potency of EBV lytic cycle reactivation induced by romidepsin is much stronger than that induced by valproic acid (5 nM romidepsin vs $5 \mathrm{mM}$ valproic acid) $[13,15]$. However, the major hindrance to the application of oncolytic therapy is the presence of refractory cell population to lytic reactivation by current lytic inducers, including HDAC inhibitors. Deciphering the mechanisms regulating the switch from viral latent to lytic cycle in cancer cells may aid in the translation of oncolytic therapy for the treatment of EBV-associated cancers.

We have been investigating the cellular consequences following EBV lytic cycle reactivation in different EBV-associated cancer cell lines. We observed that lytic 
cycle induction by HDAC inhibitors could mediate enhanced killing of EBV-positive epithelial cells when compared with their EBV-negative counterparts ${ }^{[13]}$. We further found that the enhanced killing was related to the induction of apoptosis as evidenced by the co-expression of EBV lytic proteins (e.g. Zta) and apoptotic marker (e.g. cleaved caspase-3) in EBV-positive epithelial cells upon treatment with HDAC inhibitors (e.g. SAHA) ${ }^{[14]}$. Inhibition of EBV DNA replication and expression of EBV late lytic proteins by phosphonofomic acid did not impact on SAHA's induced killing of EBV-positive epithelial cells, suggesting that induction of early phase of lytic cycle was sufficient to mediate enhanced killing of EBV-positive versus EBV-negative cancer cells. Such enhanced killing could not be observed in EBV-positive lymphoid cells (e.g. Burkitt lymphoma, NK/T cell lymphoma and lymphoblastoid cell lines) since they are not responsive to lytic cycle reactivation by HDAC inhibitors ${ }^{[13]}$. In these non-inducible lymphoid cells, we are developing other strategies to target the function of EBV latent proteins (e.g. EBV nuclear antigen-3A/-3C) $[18]$.

Several candidate viral lytic proteins might be involved in the killing of EBV-associated cancers upon treatment with HDAC inhibitors. For instance, the major EBV lytic cycle initiator Zta, which can induce phosphorylation of p53 and G2/M arrest, might directly cause cell death following lytic induction by HDAC inhibitors ${ }^{[19,20]}$. Another lytic initiator Rta can also induce irreversible G1 arrest, cellular senescence and apoptosis in EBV-positive epithelial cancer cell lines ${ }^{[21,22,23]}$. Other early viral proteins such as the EBV alkaline exonuclease BGLF5, which can shut off important cellular gene functions and trigger apoptosis, might also be involved ${ }^{[24]}$. It will be interesting to investigate whether HDAC inhibitors can modulate the function of BHRF1, an EBV-encoded early lytic protein homologous to the anti-apoptotic protein $\mathrm{Bcl}-2$, in the $\mathrm{EBV}$-positive cancer cell lines ${ }^{[25-27]}$. Another possible mechanism of action of HDAC inhibitors may be the direct acetylation of cellular tumor suppressor protein, p53, which is known to interrupt the anti-apoptotic activities of Bcl-2 family members ${ }^{[28]}$. The activities of the anti-apoptotic EBV miR-BARTs and their mRNA targets (e.g. CASZ1, DICE1 and TP53INP1) in the NPC and GC cells upon treatment with HDAC inhibitors also deserve further investigation ${ }^{[29]}$.

The roles of different HDAC isoforms (i.e., HDAC1-11) in the regulation of EBV lytic reactivation remain unclear. We showed that inhibition of HDAC-1, -2 and -3 either by selective HDAC inhibitors targeting HDAC-1, -2 and -3 (romidepsin, MS-275 or apicidin) or by shRNA knockdown is sufficient to induce EBV lytic cycle in NPC and GC cells [15]. The data are consistent with the findings that overexpression of HDAC-2 could suppress the lytic cycle reactivation in NPC cells by HDAC inhibitors ${ }^{[30]}$. Class I HDACs such as HDAC-2 and -3 are found to be important in the control of lytic reactivation of other latent viruses such as human immunodeficiency virus (HIV) and Kaposi's sarcoma-associated herpesvirus (KSHV) [31, 32]. We also observed that HDAC-2 and -3 are overexpressed in EBV-positive epithelial cells when compared with EBV-positive lymphoid cells (unpublished data). The data might explain why epithelial cells are more sensitive to the reactivation of EBV lytic cycle by HDAC inhibitors when compared with lymphoid cells ${ }^{[13,18]}$.

Several kinase pathways, including the phosphatidylinositol 3'-kinase (PI3K), MAPK/ERK kinase (MEK), c-Jun amino terminal kinase (JNK), p38 stress mitogen-activated protein kinase (MAPK), ataxia telangiectasia-mutated (ATM) kinase, and protein kinase C-delta (PKC- $\delta$ ) pathways ${ }^{[5,30,33-38]}$ were shown to control the activation of EBV lytic cycle in various cancer cell types. We demonstrated that only PKC- $\delta$, rather than PI3K, MEK, JNK, p38 MAPK, and ATM pathways, was related to the EBV lytic cycle reactivation by romidepsin ${ }^{[15]}$. The data are consistent with Tsai et al., 2011 which showed that the activation of PKC- $\delta$ and Sp1 was essential for the lytic cycle reactivation in NPC cells upon treatment with HDAC inhibitors ${ }^{[30]}$. Feng et al. also showed that activation of PKC- $\delta$ signaling was involved in the reactivation of EBV lytic cycle in GC cells by 5-FU and cisplatin ${ }^{[5]}$. However, our laboratory and others have demonstrated that the ATM pathway seems to be involved in the step of viral DNA replication rather than the initiation step of lytic cycle reactivation ${ }^{[39,40]}$, contrary to a report which showed that the activation of ATM pathway was crucial for the EBV lytic cycle reactivation [37]. Contradictory roles of PKC- $\delta$ signaling are also observed in our laboratory when we induce EBV lytic cycle in the NPC and GC cells by novel lytic inducers identified in a high-throughput screening versus HDAC inhibitors (unpublished data). The discrepant pathways of lytic reactivation suggest that resistance to EBV lytic reactivation might arise as a result of alteration of expression of various signaling molecules in different cell lines. A combination of pharmacological agents, which target multiple signaling pathways, may be required to overcome the resistance to lytic cycle reactivation in EBV-associated cancer cells.

It remains unknown whether acetylation of histone or non-histone proteins or both by HDAC inhibitors is responsible for viral lytic cycle reactivation in EBV-positive epithelial malignancies. Whilst histone acetylation was reported to be critical in the reactivation of EBV lytic cycle, some laboratories showed that histone acetylation was not 


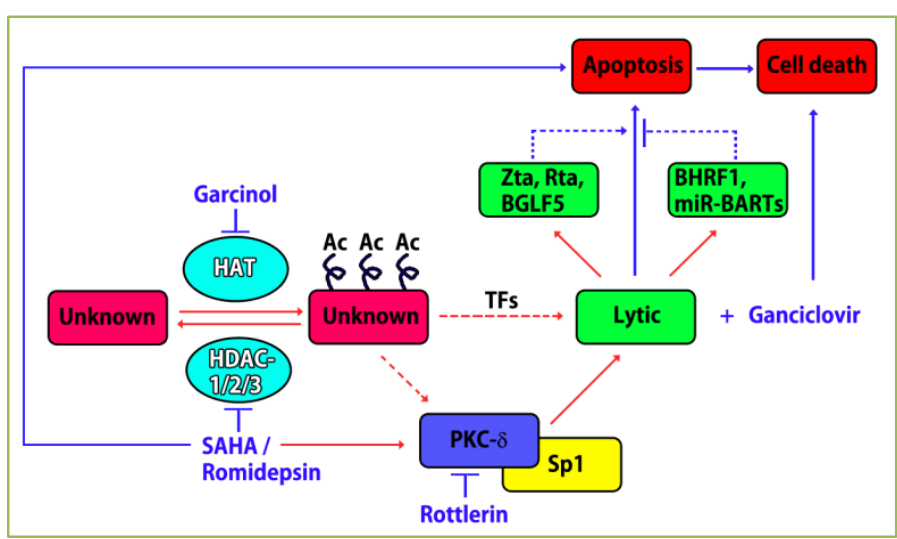

Figure 1. Schematic diagram illustrating the mechanisms of EBV lytic cycle reactivation and induction of apoptosis in EBV-positive epithelial malignancies following treatment with HDAC inhibitors. HDAC inhibitors such as romidepsin and SAHA induce EBV lytic cycle through activation of PKC- $\delta$ and acetylation of unknown histone or non-histone protein(s). HDAC inhibitors directly induce apoptosis in cancer cells and indirectly augment apoptosis of EBV-positive cancer cells through reactivation of EBV lytic cycle. Lines in red and blue colors represent pathways that may be involved in EBV lytic cycle reactivation and apoptosis, respectively. Dashed lines represent hypothetic pathways for further investigations. Ac, acetylation; TFs, transcription factors.

required ${ }^{[41-43]}$. Transcription factors, such as $\mathrm{Sp} 1, \mathrm{Sp} 3$, CREB, MEF2D, C/EBPs, ZEBs and YY1, which are known to be associated with the EBV Z promoter (the key initiator of EBV lytic cycle), can be modified by lysine acetylation [44-50]. In addition, phosphorylation of TIP60, a HAT, was found to be required for EBV DNA replication ${ }^{[39,51]}$. It will thus be interesting to study the roles of acetylation of non-histone proteins in the induction of lytic cycle of EBV. We have previously reported that a proteasome inhibitor, bortezomib, can synergize with HDAC inhibitors, including SAHA and romidepsin, to induce histone acetylation whilst it suppresses HDAC inhibitors' activation of EBV lytic cycle in NPC cells ${ }^{[52,53]}$, suggesting that induction of EBV lytic cycle may not directly correlate to the level of histone acetylation. Our current work showed that HAT inhibitors, including garcinol and anacardic acid, can significantly suppress the expression of EBV immediate-early (Zta and Rta), early (BMRF1) and late (VCA-p18) lytic proteins induced by SAHA in a dose-dependent manner (unpublished data). Interestingly, the HAT inhibitors can suppress SAHA's induction of EBV lytic cycle at a concentration that does not inhibit the histone acetylation of the $\mathrm{Z}$ promoter. On the other hand, the HAT inhibitors can abrogate SAHA's acetylation of non-histone proteins, inferring that acetylation of non-histone proteins might be involved in the regulation of EBV lytic cycle reactivation. In fact, HDAC inhibitors are shown to acetylate more than 1750 cellular proteins in different human cancer cell lines ${ }^{[12]}$. It would be interesting to identify which acetylated proteins are involved in the reactivation of EBV lytic cycle. Zp transcription factors, such as ZEBs and YY1, which can be directly acetylated by HDAC inhibitors, are potential candidates ${ }^{[49,50]}$.

Our recent work demonstrated HDAC inhibitors (e.g. SAHA and romidepsin) are potent inducers of EBV lytic cycle in EBV-positive epithelial cancer cells. Furthermore, EBV lytic cycle induction by HDAC inhibitors can mediate enhanced apoptosis of the EBV-positive cells when compared with EBV-negative cells and render EBV-positive cells susceptible to killing by GCV (Figure 1). These data support the therapeutic value of developing HDAC inhibitors as potent lytic inducing drugs for oncolytic therapy against EBV-associated epithelial malignancies. However, a certain proportion of the cancer cells is refractory to lytic cycle reactivation. In the future, we aim to further decipher the mechanisms regulating the lytic cycle reactivation in EBV-associated cancer cells. Identification of new molecules or pathways involved in EBV lytic cycle induction will open new avenues for testing and development of drugs for viral-targeted therapy against EBV-associated cancers.

\section{Conflicting interests}

The authors have declared that no competing interests exist.

\section{Acknowledgments}

The projects described in this review paper are supported by NPC Area of Excellence (AoE/M 06/08 Center for Nasopharyngeal Carcinoma Research) and EBV research (\# 200004525) grants of AKSC.

\section{Abbreviations}

EBV: Epstein-Barr virus; GCV: ganciclovir; HDAC: histone deacetylase; HAT: histone acetyltransferase; SAHA: suberoylanilide hydroxamic acid; NPC: nasopharyngeal carcinoma; GC: gastric carcinoma.

\section{References}

1. Rickinson $\mathrm{AB}$, Kieff E. Epstein-Barr virus and its replication. In: Knipe DM, Howley PW, eds. Fields virology, 5th ed. Philadelphia: Lippincott Williams and Wilkins, 2007; 2604-2654.

2. Amon W, Farrell PJ. Reactivation of Epstein-Barr virus from latency. Rev Med Virol 2004; 15:149-156.

3. Wildeman MA, Novalic Z, Verkuijlen SA, Juwana H, Huitema $\mathrm{AD}$, Tan IB, et al. Cytolytic virus activation therapy for epstein-barr virus-driven tumors. Clin Cancer Res 2012; 18:5061-5070.

4. Stoker SD, Novalic Z, Wildeman MA, Huitema AD, Verkuijlen $\mathrm{SA}$, Juwana $\mathrm{H}$, et al. Epstein-Barr virus-targeted therapy in nasopharyngeal carcinoma. J Cancer Res Clin Oncol 2015; 141:1845-1857. 
5. Feng WH, Israel B, Raab-Traub N, Busson P, Kenney SC. Chemotherapy induces lytic EBV replication and confers ganciclovir susceptibility to EBV-positive epithelial cell tumors. Cancer Res 2002; 62:1920-1926.

6. Grunstein M. Histone acetylation in chromatin structure and transcription. Nature 1997; 389:349-352.

7. $\mathrm{Xu} \mathrm{WS,} \mathrm{Parmigiani} \mathrm{RB,} \mathrm{Marks} \mathrm{PA.} \mathrm{Histone} \mathrm{deacetylase}$ inhibitors: molecular mechanisms of action. Oncogene 2007; 26:5541-5552

8. Imhof A, Yang XJ, Ogryzko VV, Nakatani Y, Wolffe AP, Ge H. Acetylation of general transcription factors by histone acetyltransferases. Curr Biol 1997; 7:689-692.

9. Munster PN, Troso-Sandoval T, Rosen N, Rifkind R, Marks PA, Richon VM. The histone deacetylase inhibitor suberoylanilide hydroxamic acid induces differentiation of human breast cancer cells. Cancer Res 2001; 61:8492-8497.

10. Klass CM, Krug LT, Pozharskaya VP, Offermann MK. The targeting of primary effusion lymphoma cells for apoptosis by inducing lytic replication of human herpesvirus 8 while blocking virus production. Blood 2005; 105:4028-4034.

11. Dokmanovic M, Clarke C, Marks PA. Histone deacetylase inhibitors: overview and perspectives. Mol Cancer Res 2007; 5:981-989.

12. Choudhary C, Kumar C, Gnad F, Nielsen ML, Rehman M, Walther T8C, et al. Lysine acetylation targets protein complexes and co-regulates major cellular functions. Science 2009; 325:834-40.

13. Hui KF, Chiang AK. Suberoylanilide hydroxamic acid induces viral lytic cycle in Epstein-Barr virus-positive epithelial malignancies and mediates enhanced cell death. Int J Cancer 2010; 126:2479-2489.

14. Hui KF, Ho DN, Tsang CM, Middeldorp JM, Tsao GS, Chiang AK. Activation of lytic cycle of Epstein-Barr virus by suberoylanilide hydroxamic acid leads to apoptosis and tumor growth suppression of nasopharyngeal carcinoma. Int J Cancer 2012; 131:1930-1940.

15. Hui KF, Cheung AK, Choi CK, Yeung PL, Middeldorp JM, Lung ML, et al. Inhibition of class I histone deacetylases by romidepsin potently induces Epstein-Barr virus lytic cycle and mediates enhanced cell death with ganciclovir. Int J Cancer 2015; doi: 10.1002/ijc.29698.

16. Harrison SJ, Bishton M, Bates SE, Grant S, Piekarz RL, Johnstone $\mathrm{RW}$, et al. A focus on the preclinical development and clinical status of the histone deacetylase inhibitor, romidepsin (depsipeptide, Istodax((R))). Epigenomics 2012; 4:571-589.

17. Piekarz RL, Frye R, Prince HM, Kirschbaum MH, Zain J, Allen $\mathrm{SL}$, et al. Phase 2 trial of romidepsin in patients with peripheral T-cell lymphoma. Blood 2011; 117:5827-5834.

18. Hui KF, Leung YY, Yeung PL, Middeldorp JM, Chiang AK. Combination of SAHA and bortezomib up-regulates CDKN2A and CDKN1A and induces apoptosis of Epstein-Barr virus-positive Wp-restricted Burkitt lymphoma and lymphoblastoid cell lines. Br J Haematol 2014; 167:639-650.

19. Mauser A, Saito S, Appella E, Anderson CW, Seaman WT, Kenney S. The Epstein-Barr virus immediate-early protein BZLF1 regulates p53 function through multiple mechanisms. J Virol 2002; 76:12503-12512.

20. Mauser A, Holley-Guthrie E, Simpson D, Kaufmann W, Kenney
S. The Epstein-Barr virus immediate-early protein BZLF1 induces both a G(2) and a mitotic block. J Virol 2002; 76:10030-10037.

21. Feng WH, Westphal E, Mauser A, Raab-Traub N, Gulley ML, Busson $\mathrm{P}$, et al. Use of adenovirus vectors expressing Epstein-Barr virus (EBV) immediate-early protein BZLF1 or BRLF1 to treat EBV-positive tumors. J Virol 2002; 76:10951-10959.

22. Wang L, Shan L, Lo KW, Yin J, Zhang Y, Sun R, et al. Inhibition of nasopharyngeal carcinoma growth by RTA-expressing baculovirus vectors containing oriP. J Gene Med 2008; 10:1124-1133.

23. Chen YJ, Tsai WH, Chen YL, Ko YC, Chou SP, Chen JY, et al Epstein-Barr Virus (EBV) Rta-Mediated EBV and Kaposi's Sarcoma-Associated Herpesvirus Lytic Reactivations in 293 Cells. PLoS One 2011; 6:e17809.

24. Zuo J, Thomas W, van Leeuwen D, Middeldorp JM, Wiertz EJ, Ressing ME, et al. The DNase of gammaherpesviruses impairs recognition by virus-specific $\mathrm{CD} 8+\mathrm{T}$ cells through an additional host shutoff function. J Virol 2008; 82:2385-2393.

25. Foghsgaard L, Jaattela M. The ability of BHRF1 to inhibit apoptosis is dependent on stimulus and cell type. J Virol 1997; 71:7509-7517.

26. Kvansakul M, Wei AH, Fletcher JI, Willis SN, Chen L, Roberts AW, et al. Structural basis for apoptosis inhibition by Epstein-Barr virus BHRF1. PLoS pathogens 2010; 6:e1001236.

27. Liu MY, Shih YY, Li LY, Chou SP, Sheen TS, Chen CL, et al. Expression of the Epstein-Barr virus BHRF1 gene, a homologue of Bcl-2, in nasopharyngeal carcinoma tissue. J Med Virol 2000; 61:241-250.

28. Yamaguchi H, Woods NT, Piluso LG, Lee HH, Chen J, Bhalla $\mathrm{KN}$, et al. p53 acetylation is crucial for its transcription-independent proapoptotic functions. J Biol Chem 2009; 284:11171-11183.

29. Kang D, Skalsky RL, Cullen BR. EBV BART MicroRNAs Target Multiple Pro-apoptotic Cellular Genes to Promote Epithelial Cell Survival. PLoS pathogens 2015; 11:e1004979.

30. Tsai PF, Lin SJ, Weng PL, Tsai SC, Lin JH, Chou YC, et al. Interplay between PKCdelta and $\mathrm{Sp} 1$ on histone deacetylase inhibitor-mediated Epstein-Barr virus reactivation. J Virol 2011; 85:2373-2385.

31. Barton KM, Archin NM, Keedy KS, Espeseth AS, Zhang YL, Gale J, et al. Selective HDAC inhibition for the disruption of latent HIV-1 infection. PLoS One 2014; 9:e102684.

32. Shin HJ, DeCotiis J, Giron M, Palmeri D, Lukac DM. Histone deacetylase classes I and II regulate Kaposi's sarcoma-associated herpesvirus reactivation. J Virol 2014; 88:1281-1292.

33. Feng WH, Hong G, Delecluse HJ, Kenney SC. Lytic induction therapy for Epstein-Barr virus-positive B-cell lymphomas. J Virol 2004; 78:1893-1902.

34. Fahmi H, Cochet C, Hmama Z, Opolon P, Joab I. Transforming growth factor beta 1 stimulates expression of the Epstein-Barr virus BZLF1 immediate-early gene product ZEBRA by an indirect mechanism which requires the MAPK kinase pathway. J Virol 2000; 74:5810-5818.

35. Adamson AL, Kenney SC. Rescue of the Epstein-Barr virus BZLF1 mutant, Z(S186A), early gene activation defect by the BRLF1 gene product. Virology 1998; 251:187-197.

36. Bryant H, Farrell PJ. Signal Transduction and Transcription Factor Modification during Reactivation of Epstein-Barr Virus from 
Latency. J Virol 2002; 76:10290-10298.

37. Hagemeier SR, Barlow EA, Meng Q, Kenney SC. The cellular ataxia telangiectasia-mutated kinase promotes epstein-barr virus lytic reactivation in response to multiple different types of lytic reactivation-inducing stimuli. J Virol 2012; 86:13360-13370.

38. El-Guindy AS, Heston L, Endo Y, Cho MS, Miller G. Disruption of Epstein-Barr virus latency in the absence of phosphorylation of ZEBRA by protein kinase C. J Virol 2002; 76:11199-11208.

39. Li R, Zhu J, Xie Z, Liao G, Liu J, Chen MR, et al. Conserved herpesvirus kinases target the DNA damage response pathway and TIP60 histone acetyltransferase to promote virus replication. Cell Host Microbe 2011; 10:390-400.

40. Hau PM, Deng W, Jia L, Yang J, Tsurumi T, Chiang AK, et al. Role of ATM in the Formation of the Replication Compartment during Lytic Replication of Epstein-Barr Virus in Nasopharyngeal Epithelial Cells. J Virol 2015; 89:652-668.

41. Countryman JK, Gradoville L, Miller G. Histone hyperacetylation occurs on promoters of lytic cycle regulatory genes in Epstein-Barr virus-infected cell lines which are refractory to disruption of latency by histone deacetylase inhibitors. J Virol 2008; 82:4706-4719.

42. Daigle D, Gradoville L, Tuck D, Schulz V, Wang'ondu R, Ye J, et al. Valproic Acid Antagonizes the Capacity of Other HDAC Inhibitors to Activate the Epstein-Barr Virus Lytic Cycle. J Virol 2011; 85:5628-5643.

43. Jenkins PJ, Binne UK, Farrell PJ. Histone acetylation and reactivation of Epstein-Barr virus from latency. J Virol 2000; 74:710-720.

44. Braun H, Koop R, Ertmer A, Nacht S, Suske G. Transcription factor Sp3 is regulated by acetylation. Nucleic Acids Res 2001; 29:4994-5000.

45. Cesena TI, Cui TX, Subramanian L, Fulton CT, Iniguez-Lluhi JA,
Kwok RP, et al. Acetylation and deacetylation regulate CCAAT/enhancer binding protein beta at K39 in mediating gene transcription. Mol Cell Endocrinol 2008; 289:94-101.

46. Gachon F, Devaux C, Mesnard JM. Activation of HTLV-I transcription in the presence of Tax is independent of the acetylation of CREB-2 (ATF-4). Virology 2002; 299:271-278.

47. Gregoire S, Xiao L, Nie J, Zhang X, Xu M, Li J, et al. Histone deacetylase 3 interacts with and deacetylates myocyte enhancer factor 2. Mol Cell Biol 2007; 27:1280-1295.

48. Waby JS, Chirakkal H, Yu C, Griffiths GJ, Benson RS, Bingle $\mathrm{CD}$, et al. $\mathrm{Sp} 1$ acetylation is associated with loss of DNA binding at promoters associated with cell cycle arrest and cell death in a colon cell line. Mol Cancer 2010; 9:275.

49. Zhao LJ, Subramanian T, Chinnadurai G. Changes in C-terminal binding protein 2 (CtBP2) corepressor complex induced by E1A and modulation of E1A transcriptional activity by CtBP2. J Biol Chem 2006; 281:36613-36623.

50. Yao YL, Yang WM, Seto E. Regulation of transcription factor YY1 by acetylation and deacetylation. Mol Cell Biol 2001; 21:5979-5991.

51. Hagemeier SR, Barlow EA, Meng Q, Kenney SC. The cellular ataxia telangiectasia-mutated (ATM) kinase promotes Epstein-Barr virus (EBV) lytic reactivation in response to multiple different types of lytic-inducing stimuli. J Virol 2012; 86:13360-13370.

52. Hui KF, Lam BH, Ho DN, Tsao SW, Chiang AK. Bortezomib and SAHA synergistically induce ROS-driven caspase-dependent apoptosis of nasopharyngeal carcinoma and block replication of Epstein-Barr virus. Mol Cancer Ther 2013; 12:747-758.

53. Hui KF, Chiang AK. Combination of proteasome and class I HDAC inhibitors induces apoptosis of NPC cells through an HDAC6-independent ER stress-induced mechanism. Int J Cancer 2014; 135:2950-2961. 